\title{
A Review of Indoor Channel Modeling Techniques for Visible Light Communications
}

\author{
A.M. Ramirez-Aguilera, J.M. Luna-Rivera \\ Dept. of Science \\ Autonomous University of San Luis Potosi \\ San Luis Potosi, Mexico \\ atziry.ra@uaz.edu.mx,mlr@fciencias.uaslp.mx
}

\author{
V. Guerra, J. Rabadan, R. Perez-Jimenez \\ IDeTIC \\ University of Las Palmas de Gran Canaria \\ Las Palmas de Gran Canaria, Spain \\ \{vguerra,jrabadan,rperez\}@idetic.eu
}

\author{
F.J. Lopez-Hernandez \\ CeDInt \\ $U P M$ \\ Madrid, Spain \\ dxtn@tfo.upm.es
}

\begin{abstract}
Visible light communications (VLC) is a technology that uses the unregulated electromagnetic spectrum band between 380 and $780 \mathrm{~nm}$ which offers an enormous potential of high speed communications for short distances, regardless of radio interference. VLC technology has many useful applications, including smart lighting, high speed data link, communication in hazardous environments, vehicular communications, underwater communications, location based services, etc. Although there is a growing interest on VLC applications, there is still a lack of proper VLC channel models. In this paper, we present a comprehensive survey of modeling techniques for visible light communications with a focus on indoor wireless optical channels. For a better understanding, we select some relevant channel models and compare their characteristics from different aspects.
\end{abstract}

\section{INTRODUCTION}

The rapidly growing use of wireless communication systems, the strict demand for quality of service (QoS) and the emergence of fifth generation (5G) wireless systems, have brought about the exploration of new alternatives in the use of the radio spectrum such as the case of the optical spectrum [1]. There are currently two identified optical spectrum bands that can be used in indoor wireless communication: the infrared (IR) and the Visible Light spectrum bands [2]. The development of LED technology has produced great expectations that have motivated different research and business groups to develop and standardize Visible Light Communication (VLC) [3]-[5]. The purpose of VLC is to add new capacities to the LED-based lamps, as information emitting devices, without losing their basic function as illumination lamps. Although both optical and Radio-Frequency (RF) communications employ electromagnetic radiation as the information medium, these concepts differ significantly in their properties. The RF channel is generally used for long distance applications where user mobility exists and there is electromagnetic radiation through obstacle and walls. Meanwhile, the optical channel is convenient for short distance applications where high capacity and speed of transmission as well as low cost and practical receivers are required. Currently, there are prospective application scenarios that are being investigated using VLC technology, for example, object and people localization [6], [7], vehicle to vehicle communication [8], underwater commu- nication [9], underground mining tunnels communication [10], [11] information displaying signboards [12], communications in restricted places (hospitals, aircrafts, etc.), Li-Fi [13], among others [14]. Within the main challenges of the optic channel is the dispersion effects caused by the multipath that produce intersymbol interference (ISI) and reduction of transmission rate. For this reason, the efficient design and implementation of the VLC systems require first to characterize the propagation conditions of the channel. During the last few years, different channel models have been developed to estimate impulse response for indoor optical wireless systems. These approaches are based on estimating the total channel impulse response model, i.e. the line-of-sight (LOS) response, where the path between emitter and receiver is unobstructed, and the multipath propagation response (NLOS), where reflections from objects (such as walls) are considered. It is important to point out that VLC channel modeling is initially based on the proposed channel models for IR, nevertheless, there are some relevant differences between them that need to be considered. Other relevant aspects, which are less explored in the literature, are the practical conditions (objects and furniture) and the illumination infrastructure of the scenarios. In addition, VLC channel modeling is expected to reliably capture the multipath propagation produced when light impinges on different surfaces and the movement of people within the scenario. Therefore, it is necessary to develop a low-complex computational channel model that considers all the parameters previously listed.

The growing application of wireless optical communications in different sectors has increased interest in the acquisition of further knowledge about the characteristics of propagation in the channel, a key aspect for the design and development of VLC systems. Thus, this article aims to present a compact and comprehensive state-of-the-art of the most relevant indoor optical channel models. As part of this review, we identify and compare the existing optical channel models methodologies. This paper is organized as follows: section II introduces the parameters of a VLC system, in section III, we provide a state-of-the-art review on the evolution of channel models. In section IV, the challenges currently facing the above-described models are presented and finally the conclusions are drawn in section V. 


\section{VLC Channel Parameters}

In the literature, many efforts have been carried out to study indoor VLC channels. In general, these works use the channel impulse response function, $h(t)$, to study the VLC propagation channel characteristics. For a given scenario, a generic propagation model can be expressed as:

$$
h(t)=h^{0}(t)+\sum_{k=1}^{\infty} h^{k}(t)
$$

where $h^{0}(t)$ defines the LOS impulse response and the second term of the equation describes the undergoing reflections in the environment of interest. The two most typical parameters used to describe the channel models are [15]:

i) Direct Current (DC) Channel Gain. The frequency response of the VLC channel has a relatively flat aspect in the area of low frequencies. In most cases, the value of the amplitude of channel direct current gain $(H(0))$, is used to characterize the losses in the channel, which relates the average optical power received, $P_{r}$, and transmitted, $P_{t}$, as follows

$$
P_{r}=H(0) P_{t}
$$

where $H(0)=\int_{-\infty}^{\infty} h(t) d t$.

ii) RMS delay spread. The received optical signal in a VLC system is subject to the multipath propagation loss in the channel. The root-mean-square (RMS) delay spread measures the temporal dispersion caused by multipath channel it also allows to obtain an estimate of the intersymbol interference. The RMS delay spread $\left(\sigma_{\tau}\right)$ is defined as

$$
\sigma_{\tau}=\sqrt{\frac{\int_{-\infty}^{\infty}(t-\tau)^{2} h^{2}(t) d t}{\int_{-\infty}^{\infty} h(t) d t}}
$$

where $\tau$ denotes the mean excess delay given as

$$
\tau=\frac{\int_{-\infty}^{\infty} t \dot{h}^{2}(t) d t}{\int_{-\infty}^{\infty} h^{2}(t) d t} .
$$

More recently, other related features of the VLC channels have been studied such as signal bandwidth, coverage maps, wavelength dependence characteristics, etc. However, it is necessary to consider the features of the application scenario when calculating the VLC channel impulse response.

Unlike most communication systems, a VLC link is not designed exclusively for data transmission but also for illumination. Focusing primarily on the communication system criteria, such as maximum data rates, a channel model represent a useful tool to estimate a communication link. Considering the different channel models that have been proposed for indoor optical wireless communication, it is possible to establish the following characteristics that a realistic channel model for VLC must fulfill.

1) To consider the broad emission spectrum (band of visible light) of the LED's additional to emission patterns.
In turn, it is important to consider their position and orientation within the scenario.

2) To include the objects and the multiple lamps infrastructure of typycal indoor scenarios.

3) It must be able to calculate reflections according to the type of surface. In addition, the value of reflectance for each component of the wavelength must be considered. It should also be able to analyze the shadow effects produced by the objects in the scenario.

4) To consider the possibility of movement of objects within the scenario.

5) All these previously mentioned parameters must be modeled with a low computational complexity.

Therefore, the use of models that allow the characterization of a channel that is closer to reality will be favorable to, to a higher degree, the evolution and implementation of VLC technology. As there is no simple analytic calculation method for this problem, computationally efficient simulation algorithms are required. Despite this increasing attention on VLC systems, there is a lack of proper visible light channel models. This is a serious concern since channel modeling is the very first step for efficient, reliable, and robust VLC system design. In the following section we present a revision of the state-of-the-art in channel modeling for indoor VLC systems.

\section{STATE-OF-THE-ART}

In this section, we present a review of the leading research related to modeling and characterization for the indoor optical wireless communication channel. This review highlights the most relevant techniques used in the literature to evaluate the impulse response of the optical wireless channels. However, and despite the differences between VLC and infrared channel modeling, many of these techniques have been extended from the models reported in the past on infrared channel modeling. Therefore, we consider the description of early VLC channel models, based on infrared communications, essential to understand the state-of-the-art in channel modeling for VLC so far.

\section{A. Indoor Optical Wireless Channel Models}

In [16], Gfeller and Bapst presented the first analytical model for the characterization of indoor optical communication channels in the infrared spectrum. They proposed to calculate the received power by means of a numerical integration of power contributions from the surface differentials of the given scenario. These results were limited to one reflection since the number of calculations increased at an exponential rate with the number of reflections. Given that this analysis was insufficient to determine the channel temporary dispersion and bandwidth, there was a need to develop alternative methods to capture these propagation features for a higher number of reflections with lower computational cost. A new model was proposed by Barry in [17] as an alternative to Gfeller's analytical model. In this model, each surface of the room (walls, ceiling, floor, and objects) is divided into small reflecting surfaces characterized by their area and reflection coefficient. 
Each small reflecting surfaces is considered as a receiver of the LOS component emitted from the transmitter; then it becomes an individual transmitter whose output signal depends on the incident ray power, and the reflection coefficient of the surface; as a result, the first reflection is defined by the total LOS power received from each small reflecting surfaces. For the second reflection, each small reflecting surfaces is considered as a new transmitter that contributes in each of the remaining small reflecting surfaces; its received power is calculated similar to the first reflection. The total contributions in the receiver define the value of the second reflection. This procedure is repeated in function of the number of reflections to be analyzed. This method becomes more precise if the size of the small reflecting surfaces is reduced; nevertheless, its complexity has a fast exponential growth because of the number of reflections and the total number of small reflecting surfaces.

Carruthers and Kannan proposed the execution of Barry's recursive algorithm as an iterative algorithm and the computation time is considerably reduced [18]. In a later version [19], the iterative approach was modified to include multiple transmitters and receivers, as well as including reflecting surfaces such as furnitures in addition to walls, floor, and ceiling. Similarly, Abtahi and Hashemi use Barry's recursive algorithm to analyze the shadowing effects due to objects in the environment [20]. Lopez-Hernandez et. al. implemented a method to reduce the computation time of Barry's original algorithm when the number of reflections are increased [21]. This approach divides the simulation time into intervals, instead of reflections, in this way partial results of the simulation are first stored in matrices and used in subsequent time intervals of the simulation. This strategy had the advantage of simulating several transmitters and receivers jointly within the room, which allowed the study of different types of distortions such as intersymbol interference. A downside of this methodology is the large amount of memory utilized to store these matrices.

Despite of the improvements made to Barry's model, the existing approaches still required a very high computational cost to provide the propagation characteristics of practical optical communication channels. In addition to this complexity problem, all Barry's based models consider only the existence of diffuse reflectors which is a limitation for real scenarios. In this way, another type of iterative channel modeling techniques, that combine ray tracing method with Monte Carlo simulation were proposed to estimate the channel impulse response of indoor optical communication systems. LopezHernandez et al. presented for the first time in [22] the characterization of an indoor wireless optical channel by combining Ray Tracing technique with Monte Carlo simulations. In this approach, the optical power of the transmitter is represented in the form of rays which propagate following straight line paths at the speed of light. Every time that a ray is reflected on a surface, it will deviate towards a new direction and its transmitted power will depend on the characteristics of the surface. In this method, there is no limitation on the number of reflections, however, it requires to generate a very high number of rays due to the low probability of a ray reaching the receiver. In a later work, the same authors introduced the Modified Monte Carlo and Ray Tracing method (MMC) [23], which is an improvement of the original technique, it basically assumes that the rays in each point of reflection contribute in the receiver with a LOS component. With this consideration, the MMC reduces significantly the number of rays required to estimate the optical channel impulse response as compared to the method published in [22]. MMC was first applied to the infrared spectrum and later extended to the visible light spectrum [24].

After the introduction of the MMC method, further investigations were carried out to model different type of transmitters, receivers, as well as reflecting surfaces. Among these works, based on the MMC, we can highlight [25]-[29]. Zhang et al. proposed in [25] an algorithm that lessen the number of rays as the number of reflections grows which also decreases the computational complexity of the MMC. The authors assume that the probability that a ray is reflected, once it has impinged on a surface, depends on the reflection coefficient $(\rho)$. Furthermore, Rodriguez et al. incorporated in [26] the use of the Phong reflection model, allowing the characterization of specular and/or diffuse surfaces with the MMC algorithm. This type of models makes it possible to characterize surfaces with high accuracy. In [27], the author provides a similar way for calculating impulse response. It assumes a photon emission model and the propagation of the light wave is described using the ray tracing technique. Once a ray hits a reflecting surface, the direction is described by the Bidirectional Reflection Distribution Function of each surface. In [28], Mavrakis et al. proposed a geometrical propagation model based on the MMC model, where the reflecting points are obtained in a first instance and then used to calculate the subsequent reflection points, instead of dividing the surrounding surfaces into small reflecting elements. The computational time for this algorithm is similar to the proposed by Barry's model. More recently, Chowdhury et al. suggested in [29] to combine the reflecting cells' model with MMC. In order to calculate the first reflection, the surfaces are divided into small reflecting surfaces, then the MMC is applied for the subsequent reflections. The results show that this approach yields a good approximation to estimate the impulse channel response, since the first reflection contributes with a high percentage of the total diffuse power received. However, the time of execution is still high and its computational complexity depends on the room dimensions [30].

In [31], the authors propose a statistical channel model based on a mathematical expression to estimate the dispersion parameters of the optical channel. This approach employs some variables to define both the environment and the transmitter and receiver configurations. The dispersion parameters of the channel impulse response are then obtained using the Rayleigh and Gamma distribution functions. However, as a drawback of this strategy is the little flexibility when conditions in the environment and/or configurations of transmitter and receiver are altered. Thus, if an element of the scenario 
changes, it is necessary to estimate again the total channel impulse response.

In [32] Jungnickle et al. provide a simple model for indoor unguided optical communications for infrared spectrum. This model is based on the Ulbricht integrating sphere model to estimate the channel impulse response in the frequency domain using the Rician distribution function and the room parameters.

\section{B. VLC Channel Models}

Much of the work that has been done on channel characteristics for indoor infrared communication have been adopted or extended to VLC. However, it is important to emphasize the differences between the visible light and infrared bands. As opposed to communication in the infrared spectrum, the LED emission power is distributed over a wider range of wavelengths in the visible light spectrum where the channel conditions yield by the reflecting coefficients for each surface are a function of the wavelength. Along with this, it is possible that other phenomena different to the reflection appear and it represents changes in the impulse response as a function of the wavelength. Furthermore, VLC aims to use light sources for lighting and communication then it is necessary to consider the physical characteristics of commercial LED devices as well as their parameters such as radiation pattern, orientation, power emission and their space distribution in the room. Moreover, furnitures, windows or industrial areas should also be incorporated along with the type of material they are made such as metallic, plastic and polymeric surfaces. All these elements must be considered in order to estimate the VLC channel in a more realistic way. Next, we describe some of the research works addressing these modeling issues in order to provide with a more complete optical wireless channel model for VLC.

In [33], the authors provide a VLC channel model, based on the model proposed in [32], considering the wide emission spectrum of the LED but where the reflectance value of the surfaces remains as a constant. In a similar way, Nguyen et al. and Komine and Nakagawa in [34] and [35] use Barry's method, considering also a LED as the transmitter, however, the wavelength dependence of the reflectance is also ignored. In [15], Long et al. include the reflectance information using the reflectance mean value of the whole spectrum of visible light for each surface. Nevertheless, the first attempt at characterizing the VLC channel with different values of reflectance was yielded by Lee et al. in [36]. Lee's model is based on the Barry's model that analyzes the signal components in the visible light spectrum with a white phosphorus LED spectrum. It uses the mean value for calculating the channel impulse response as function of the wavelength. The authors make a distinction between infrared and Light Visible Channel and claim that the total diffuse power and delay spread are smaller in VLC than infrared. These results are assuming an empty room where the surfaces are completely diffuse.

In [37] a tool based on MMC algorithm for simulating the channel impulse response for indoor VLC using 3D CAD models was presented. The simulation tool developed allows to evaluate the VLC channels and the 3D simulation environment can be defined using any CAD software capable of generating $3 \mathrm{D}$ vector-type graphics and storing them in a 3D-format file.

More recently, a channel model based ray tracing is proposed in [38] using the illumination design software Zemax ${ }^{\circledR}$. This tool creates a tridimensional simulation environment allowing to define the geometry of the room, the reflection characteristics of the materials of the surface, the specifications of the light sources and the receivers. This model also allows to incorporate objects and defines the reflection coefficient as a function of the wavelength for each material on the surface. The CAD software uses the method of Ray Tracing and Monte Carlo method for calculating the channel impulse response, and in turn, employs an optimized sampling method for generating the rays. The model, however, is limited to the analysis of the incidence effect that rays have on surfaces, that represent a change of energy in the wavelength spectrum, which are currently present in different objects. In [5], Uysal et al. presents four reference scenarios built with Zemax ${ }^{\circledR}$ to characterize the VLC channel that have been adopted by IEEE 802.15.7r1. These scenarios are described below:

a) A workplace with dimensions $(14 \mathrm{~m} \times 14 \mathrm{~m} \times 3 \mathrm{~m})$ including wooden floor, plastered walls and ceiling. Wooden desks with chairs are distributed in the scenario. There are 32 LED luminaries distributed on the ceiling of 73 $\mathrm{lum} / \mathrm{W}$ that yields an average illumination of 533 lux.

b) An office room with dimensions $(5 \mathrm{~m} \times 5 \mathrm{~m} \times 3 \mathrm{~m})$ with wooden floor, plastered walls and ceiling. There are two luminaries, one on the ceiling and the other on a wooden desk where there is a bright black desktop computer as a receiver. The scenario includes a window and furnitures such as bookcase, sofa and table.

c) A living room with dimensions $(6 \mathrm{~m} \times 6 \mathrm{~m} \times 3 \mathrm{~m})$ in which 9 LED luminaries of $67 \mathrm{lum} / \mathrm{W}$ are distributed. The scenario is furnished with a dining table and a set of four chairs, a sofa and a coffee table.

d) The manufacturing cell is an area where a robotic arm is located in a space of $(8.03 \times 9.45) \mathrm{m}^{2}$ with a height of $6.8 \mathrm{~m}$. To protect the area, a $1 \mathrm{~m}$-high folding screen composed of metal and glass is used to surround a robot. The transmitters are located on each screen of the protecting area surrounding the robot.

For these scenarios different reception points are assessed at tabletop height, at ear level, and at mid-body height of people standing or sitting. A detailed explanation of these location points as well as the simulated scenarios can be consulted in [5].

In [39], [40], Ramirez et al. proposed the Monte Carlo Matrix Model and Ray Tracing (M-MMC) technique. Based on the MMC approach, this technique allows the analysis of the channel impulse response as a function of the wavelength with a simplified matrix model. It also provides the framework to analyze propagation phenomena implying changes on wavelength such as when light impinges on transparent and opaque objects. In [41], the authors investigate different 
TABLE I

Summary of INDOOR Channel MOdEling TeChNiQues For Visible LIGHT COMMUNICATIONS

\begin{tabular}{|l|l|l|l|}
\hline Feature & M-MMC [39] & Lee [36] & Uysal [38] \\
\hline \hline $\begin{array}{l}\text { Emission } \\
\text { Spectrum }\end{array}$ & Polychromatic & Polychromatic & Polychromatic \\
\hline $\begin{array}{l}\text { Radiation Pattern } \\
\text { of the Light } \\
\text { Source }\end{array}$ & Adaptive & Lambertian & $\begin{array}{l}\text { Realistic light } \\
\text { source }\end{array}$ \\
\hline $\begin{array}{l}\text { Type of Propaga- } \\
\text { tion Phenomena } \\
\text { Analyzed }\end{array}$ & $\begin{array}{l}\text { Reflection, } \\
\text { Refraction, } \\
\text { Absorption }\end{array}$ & Reflection & Reflection \\
\hline $\begin{array}{l}\text { Surface } \\
\text { Reflection Model }\end{array}$ & $\begin{array}{l}\text { Lambertian, } \\
\text { Phong (Phong- } \\
\text { retroreflective, } \\
\text { Blinn, } \\
\text { Lafortune) }\end{array}$ & Lambertian & $\begin{array}{l}\text { Specular and } \\
\text { Diffuse }\end{array}$ \\
\hline $\begin{array}{l}\text { Scenario Repre- } \\
\text { sentation Tool }\end{array}$ & CAD [37] & Box Model & Zemax ${ }^{(B)}$ \\
\hline $\begin{array}{l}\text { Computational } \\
\text { complexity }\end{array}$ & Low & High & Not Available \\
\hline
\end{tabular}

bidirectional reflectance distribution function models which include Lambert, Phong, Blinn and Lafortune distributions to characterize more realistic surfaces of an indoor wireless optical channel using the MMC technique. The results show that the channel delay spread and bandwidth vary according to the selected reflection model.

More recently, an important number of works have been emerged in the literature for VLC, however, they are mainly oriented to the analysis of VLC application rather than introducing novel improvements to the channel modeling problem [7], [42]-[45]. In general, these works assume some sort of simplifications to evaluate the propagation channel such as only LOS component considerations, ideal Lambertian source, purely diffuse reflections, and/or fixed reflectance values for surface materials. For instance, the authors in [45] use the model from [5] to characterize the mobility of a receiver, a cellular phone that moves through different trajectories. We conclude this section with Table I that summarizes the most representative indoor channel model techniques for Visible Light Communications so far.

\section{Challenges}

As the design and evaluation of transmission schemes depend on the knowledge of the propagation channel, it is clear that the characterization and modeling of light propagation for VLC in realistic indoor scenarios still represent an important research challenge. Channel modeling has become an essential element of wireless communications standards including IEEE 802.15.7. The task group 802.15.7r1 suggests the use of a commercial software for lighting design so as to simulate more realistic scenarios. The results obtained with this type of tools, as well as the models proposed in the literature so far, are limited to characterize the temporal dispersion of the channel considering only the phenomenon of reflection, leaving aside other propagation phenomena such as the refraction and absorption yielded by common objects.
However, we remark that a complete analysis of the VLC channel must consider most of the phenomena that occur when light affects different types of surfaces, including the distribution of power in the broad spectrum of visible light. In addition, it is desirable that simulation tools can provide a full characterization of the channel with the lowest possible computational complexity. Moreover, people moving within the scenario is a little explored area that should be paid attention.

\section{Conclusions}

The study of the communication channel represents a key element for the design and development of VLC systems. In this sense, this paper provided a comprehensive survey on propagation channel models for VLC. Here, we reviewed, analyzed and compared the main contributions related to the estimation problem of the channel impulse response for indoor scenarios.

Although, many channel models in the past have been based on IR channel modeling, it is important to note that there exist significant differences between VLC and IR communications that avoids the application of those results to VLC channel modeling in a straightforward manner. For example, a white light LED source is fundamentally polychromatic, i.e. wideband, while an IR source can be considered as a near monochromatic emitter. In the VLC channel, the reflection coefficients of the materials have a different value for each wavelength component, unlike the IR spectrum where the reflection coefficient is constant. Furthermore, the phenomena that occur when light affects different types of surfaces should be also taken into consideration due to the practical nature of VLC scenarios. Therefore, one of the main challenges of VLC channel modeling is to develop a comprehensive method with the capability of, among other things, estimating the channel impulse response as a function of wavelength, incorporate the objects that describe a more realistic scenario, as well as consider other propagation phenomena different to reflection. On top of that, it is desirable to study the VLC channel under a realistic illumination set up that combines installation styles, light fixtures, architectural aesthetics and human physiology, the foregoing in the least amount of time possible. Moreover, channel measurements are also needed to simulate and design VLC systems. Finally, the presence of moving people and/or elements that change position also makes the measurements of VLC signals in real propagation environments a current challenge for the research community.

\section{REFERENCES}

[1] S. Wu, H. Wang, and C. Youn, "Visible light communications for 5G wireless networking systems: from fixed to mobile communications," IEEE Network, vol. 28, no. 6, pp. 41-45, 2014.

[2] Z. Ghassemlooy, S. Arnon, M. Uysal, Z. Xu, and J. Cheng, "Emerging Optical Wireless Communications-Advances and Challenges," IEEE J. on Selec. Areas in Commun., vol. 33, no. 9, pp. 1738-1749, 2015.

[3] "Visible Light Communications Consortium (VLCC)," http://www.vlcc.net, 2013, [Online].

[4] "IEEE Standard for Local and Metropolitan Area Networks-Part 15.7: Short-Range Wireless Optical Communication Using Visible Light,' IEEE Std 802.15.7-2011, pp. 1-309, Sept 2011. 
[5] M. Uysal, F. Miramirkhani, O. Narmanlioglu, T. Baykas, and E. Panayirci, "IEEE 802.15.7.r1 Reference Channel Models for Visible Light Communications," IEEE Communic. Magazine, vol. 55, no. 1, pp. 212-217, 2017.

[6] W. Zhang, M. Chowdhury, and M. Kavehrad, "Asynchronous indoor positioning system based on visible light communications," Optical Engineering, vol. 53, no. 4, pp. 1-10, 2014.

[7] J. Ding, Z. Xu, and L. Hanzo, "Accuracy of the point-source model of a multi-led array in high-speed visible light communication channel characterization," IEEE Photonics Journal, vol. 7, no. 4, pp. 1-14, 2015.

[8] Z. Ghassemlooy, S. Arnon, M. Uysal, Z. Xu, and J. Cheng, "Demostration of vehicular visible light communication based on LED headlamp," Int. Journal of Automotive Technology, vol. 17, no. 2, pp. 347-352, 2016.

[9] H. Oubei, R. ElAfandy, K. Park, T. Ng, M. Alouini, and B. Ooi, "Performance evaluation of underwater wireless optical communications links in the presence of different air bubble populations," IEEE Photonics Journal, vol. 9, no. 2, pp. 1-9, 2017.

[10] D. Iturralde, F. Seguel, I. Soto, C. Azurdia, and S. Khan, "A new vlc system for localization in underground mining tunnels," IEEE Latin America Transactions, vol. 15, no. 4, pp. 581-587, April 2017.

[11] F. Seguel, I. Soto, P. Adasme, N. Krommenacker, and P. Charpentier "Potential and challenges of vlc based ips in underground mines," in 2017 First South American Colloquium on Visible Light Communications (SACVLC), Nov 2017, pp. 1-6.

[12] S. Park, D. Jung, H. Shin, Y. Hyun, K. Lee, and Y. Oh, "Information broadcasting system based on visible light signboard," in International Conference on Wireless and Optical Communications, 2007, pp. 1-3.

[13] H. Haas, L. Yin, Y. Wang, and C. Chen, "What is LiFi?" Journal Lightwave Technology, vol. 34, no. 6, pp. 1533-1544, 2016.

[14] Z. Ghassemlooy, L. Alves, S. Zvanovec, and M. Khalighi, Visible Light Communications. Theory and Applications, 1st ed. CRC, 2017.

[15] S. Long, M. Khalighi, M. Wolf, S. Bourennane, and Z. Ghassemlooy, "Channel characterization for indoor visible light communications," in Internat. Workshop in Optical Wireless Communic., 2014, pp. 75-79.

[16] F. Gfeller and U. Bapst, "Wireless in-house data communication via diffuse infrared radiation," Proc. of the IEEE, vol. 67, no. 11, pp. 14741486, 1979.

[17] J. Barry, J. Kahn, W. Krause, E. Lee, and D. Messerschmitt, "Simulation of multipath impulse response for indoor wireless optical channels," IEEE J. on Selec. Areas in Commun., vol. 11, no. 3, pp. 367-379, 1993.

[18] J. Carruthers and P. Kannan, "Iterative site-based modeling for wireless infrared channels," IEEE Trans. on Antennas and Propagation, vol. 50, no. 5, pp. 759-765, 2002

[19] J. Carruthers, S. Carroll, and P. Kannan, "Propagation modelling for indoor optical wireless communications using fast multi-receiver channel estimation," IEE Proc.-Optoelec., vol. 150, no. 5, pp. 473-481, 2003.

[20] M. Abtahi and H. Hashemi, "Simulation of indoor propagation channel at infrared frequencies in furnished office environments," in IEEE Intern. Symp. on Personal, Indoor and Mobile Radio Commun. IEEE, 1995.

[21] F. Lopez-Hernandez and M. Betancor, "DUSTIN: a novel algorithm for the calculation of the impulse response on IR wireless indoor channels," Electronic Letters, vol. 33, no. 21, pp. 1804-1806, 1997.

[22] F. Lopez-Hernandez, R. Perez-Jimenez, and A. Santamaria, "Monte Carlo calculation of impulse response on diffuse IR wireless indoor channels," Electronic Letters, vol. 34, no. 12, pp. 1260-1262, 1998.

[23] F. L. Hernandez, R. Perez-Jimenez, and A. Santamaria, "Modified Monte Carlo scheme for high-efficiency simulation of the impulse response on diffuse IR wireless indoor channel," Electronic Letters, vol. 34, no. 19, pp. 1819-1821, 1998

[24] H. Chun, C. Chiang, and D. O'Brien, "Visible light communication using OLEDs: Illumination and channel modelling," in International Workshop on Optical Wireless Communications, ser. IWOW, Pisa, 2012.

[25] M. Zhang, Y. Zhang, X. Yuan, and J. Zhang, "Mathematic models for a ray tracing method and its applications in wireless optical communications," Optics Express, vol. 18, no. 17, pp. 18 431-18 437, 2010.

[26] S. Rodriguez, R. Perez-Jimenez, F. Lopez-Hernandez, O. Gonzalez, and A. Ayala, "Reflection model for calculation of the impulse response on IR wireless indoor channels using ray-tracing algorithm," Microwave and Optical Technology Letters, vol. 32, no. 4, pp. 296-300, 2002.

[27] H. Lee, "A Photon Modeling Method for the Characterization of indoor optical wireless communications," Progress In Electromagn. Research, vol. 92, pp. 121-136, 2009.
[28] D. Mavrakis and S. Saunders, "A novel modelling approach for wireless infrared links," in The Third International Symposium on Wireless Personal Multimedia Communications, 2000, pp. 609-614.

[29] M. Chowdhury, W. Zhang, and M. Kavehrad, "Combined deterministic and modified Monte Carlo method for calculating impulse responses of indoor optical wireless channels," J. of Lightwave Tech., vol. 32, no. 18, pp. 3132-3148, 2014

[30] M. Kavehrad, M. Chowdhury, and Z. Zhou, Short-Range Optical Wireless: Theory and Applications, 1st ed. John Wiley and Sons, 2016.

[31] R. Perez-Jimenez, J. Berges, and M. Betancor, "Statistical model for the impulse response on infrared indoor diffuse channels," Electronics Letters, vol. 33, no. 15, pp. 1298-1300, 1997.

[32] V. Jungnickel, V. Pohl, S. Nonnig, and C. Helmolt, "A physical model of the wireless infrared communication channel," IEEE J. on Selected Areas in Communic., vol. 20, no. 3, pp. 631-640, 2002.

[33] J. Grubor, S. Randel, K. Langer, and J. W. Walewski, "Broadband information broadcasting using LED-based interior lighting," Journal of Lightwave Technology, vol. 26, no. 24, pp. 3883-3892, 2008.

[34] H. Nguyen, J. Choi, M. Kang, Z. Ghassemlool, D. Kim, S. Lim, T. Kang, and C. Lee, "A MATLAB-based simulation program for indoor visible light communication system," in Intern. Symp. on Commun. Systems Netw. and Digital Signal Proc., 2010, pp. 537-541.

[35] T. Komine and M. Nakagawa, "Performance evaluation of visible-light wireless communication system using white LED Lightings," in Internat. Symp. on Computers and Communications, 2004, pp. 258-263.

[36] K. Lee, H. Park, and J. Barry, "Indoor channel characteristics for Visible Light Communications," IEEE Communic. Letters, vol. 15, no. 2, pp. 217-219, 2011

[37] S. Perez-Rodriguez, R. Perez-Jimenez, B. Rodriguez-Mendoza, F. Lopez-Hernandez, and A. Ayala-Alfonso, "Simulation of impulse response for indoor visible light communications using $3 \mathrm{~d}$ cad models," EURASIP Journal on Wireless Communications and Networking, vol. 2013, no. 1, p. 7, Jan 2013

[38] F. Miramirkhani and M. Uysal, "Channel modeling and characterization for visible light communications," IEEE Photonics J., vol. 7, no. 6, pp. $1-20,2015$.

[39] A. Ramirez-Aguilera, J. Luna-Rivera, V. Guerra, J. Rabadan, R. PerezJimenez, and F. Lopez-Hernandez, "Multi-wavelength modelling for vlc indoor channels using montecarlo simulation," in Intern. Symp. on Communic. Systems, Netw. and Digital Signal Proc. USA: IEEE, 2016.

[40] A. Ramirez-Aguilera, J. Luna, V. Guerra, J. Rabadan, R. Perez-Jimenez, and F. Lopez-Hernandez, "A generalized multiwavelength propagation model for VLC indoor channels using Monte Carlo simulation," Trans. on Emerging Telecomm. Technologies, p. e3490, 2018.

[41] J. Rufo, J. Rabadan, V. Guerra, and R. Perez-Jimenez, "Brdf models for the impulse response estimation in indoor optical wireless channels," IEEE Photonics Techn Letters, vol. 29, no. 17, pp. 1431-1437, 2017.

[42] Z. Vatansever and M. Brandt-Pearce, "Visible light positioning with diffusing lamps using an extended kalman filter," in IEEE Wireless Communications and Networking Conference, March 2017, pp. 1-6.

[43] R. Zhang, Y. Cui, H. Claussen, H. Haas, and L. Hanzo, "Anticipatory association for indoor Visible Light Communications: Light, follow me!" IEEE Transactions on Wireless Communications, vol. 17, no. 4, pp. 2499-2510, 2018

[44] P. Chvojka, S. Zvanovec, P. Haigh, and Z. Ghassemlooy, "Channel characteristics of Visible Light Communications within dynamic indoor environment," J. of Lightw Tech., vol. 33, no. 9, pp. 1719-1725, 2015.

[45] F. Miramirkhani, O. Narmanlioglu, M. Uysal, and E. Panayirci, "A mobile channel model for VLC and application to adaptive system design," IEEE Commun. Letters, vol. 21, no. 5, pp. 1035-1038, 2017. 